\title{
Oportunidades y amenazas que presenta el combate al COVID-19 para la geomática en Uruguay
}

\section{Opportunities and threats that the fight against COVID-19 presents for geomatics in Uruguay}

\author{
Rosario Casanova* \\ Miguel Gavirondo** \\ Eduardo Vásquez ${ }^{* * *}$
}

Fecha de recibido: 24 de julio de 2020 Fecha de aceptado: 30 de octubre de 2020

\section{Resumen}

La pandemia del COVID-19 permitió que, algunas herramientas geomáticas bien establecidas en el ámbito profesional, pudieran ser apreciadas por el público y por las autoridades de gobierno. Este nuevo posicionamiento se debe al uso de la geomática como medio de comunicación y como instrumento para el apoyo a la toma de decisiones. Hecho que es percibido como una gran oportunidad para la geomática al generar una concientización del valor de sus métodos, tecnologías y herramientas. Por otro lado, dicha exposición puede poner en evidencia ciertas debilidades o limitaciones, en lo que refiere al despliegue y aprovechamiento de su uso en todo su potencial. Tal es el caso de los análisis espaciales en los que se necesita llegar a un nivel de detalle más alto o, cuando se pone en juego la privacidad de los ciudadanos, como ocurre con las aplicaciones de seguimiento por

* Facultad de Ingeniería, Universidad de la República, Uruguay, correo electrónico: casanova@fing.edu.uy. ORCID: https://orcid.org/0000-0002-1873-207X

** Facultad de Ingeniería, Universidad de la República, Uruguay, correo electrónico: miguelg@fing.edu.uy

*** Facultad de Ingeniería, Universidad de la República, Uruguay, correo electrónico: evasquez@fing.edu.uy.ORCID: https://orcid.org/0000-0002-4623-0739

Colaboradores: Carlos Chiale, correo electrónico: cchiale@fing.edu.uy; Paula Caram, correo electrónico: pcaram@fing.edu.uy 
cercanía o que integran la ubicación precisa de los lugares con personas contagiadas.

En este artículo, se hace un breve análisis cualitativo de estos fenómenos, enfocado al caso uruguayo, se enumeran las limitantes y riesgos que acarrea esta exposición y se esbozan algunas oportunidades de mejora.

Palabras clave: herramientas geomáticas, tableros de control geográficos, geolocalización, precisión de los geodatos, calidad de la información geográfica.

\section{Abstract}

The COVID-19 pandemic has allowed geomatic tools to be appreciated by the public and by government, both as means of communication and as a relevant instrument to support decision-making.

This is a great opportunity for geomatics, due to its visibility, highlighting the value of its methods, technologies, and tools. On the other hand, this situation exposes certain weaknesses or limitations of geospatial information regarding the potentiality of its use. Some of these are related to the level of disaggregation of the data or to the privacy of citizens, as what happens with the tools of proximity monitoring and with studies about the mobility of people infected.

In this article, using the Uruguayan case, a brief qualitative analysis of these phenomena is made, the advantages and limitations are listed and some opportunities for improvement are outlined.

Key words: Geomatic tools, dashboards, geolocation, precision of geodata, quality of geographic information.

\section{Introducción}

La pandemia del COVID-19 propició, en los medios masivos de comunicación, una cobertura cuasi excluyente, desde el mes de marzo de 2020 y que se extiende a la fecha. En dicho contexto, ha sido notoria la exposición que han cobrado algunas herramientas geomáticas, como los tableros de control o de operaciones (operations dashboards), siendo el más popular de ellos, el elaborado en la Universidad Johns Hopkins, ${ }^{1}$ aunque no el único. Estas herramientas, por lo pronto bastante establecidas y aceptadas por la comunidad geomática, han cobrado una alta visibilidad para el gran público, al ser presentadas diariamente en cualquier informativo de televisión, portal de noticias web o red social con un alto número de visitas (Tribollet, 2020). 
En el contexto de Uruguay, el tablero ${ }^{2}$ del SINAE (Sistema Nacional de Emergencias), es sin duda, una de las metáforas ubicuas del estado de situación diaria del COVID-19.

En el presente artículo se realiza en un primer apartado, una evaluación cualitativa sobre la percepción general del valor de las herramientas geomáticas para la comunicación, el conocimiento y la gestión. Luego, se analizan algunas limitantes que restringen un mejor aprovechamiento de los datos geoespaciales. Posteriormente, se consideran los riesgos que dicha exposición acarrea. Finalmente, se reflexiona sobre algunas oportunidades de mejora que podrían redundar en un mejor posicionamiento de la geomática y su relevancia social.

\section{Evaluación cualitativa}

A nivel general se aprecia por parte de la ciudadanía, una valoración muy positiva del uso de las geo-herramientas en las tareas de comunicación y el conocimiento general. Esto se percibe, por ejemplo, en el requerimiento de mayor detalle en el espacio y en el tiempo de información georreferenciada (Rolnik, 2020), en la permanencia de herramientas embebidas en portales diarios, en organismos de gobierno, etc., y en su disponibilidad en múltiples plataformas (Tribollet, 2020).

En el caso particular del Uruguay, desde que los primeros casos de contagio fueron detectados -13 de marzo del 2020-, la Presidencia de la República, a través del Sistema Nacional de Emergencias (SINAE), emprendió una estrategia comunicacional basada inicialmente, en una rueda de prensa diaria y en la emisión de un informe de situación que incluye -a la fecha-, además de la información descriptiva, un tablero operacional que presenta la distribución geográfica, a nivel departamental, de los casos positivos de COVID-19, así como un conjunto de indicadores sobre el estado de la pandemia en Uruguay. Complementariamente, como estrategia masiva de comunicación se han utilizado las redes sociales, particularmente, desde el SINAE y Comunicación de Presidencia se publican permanentemente anuncios en Twitter referentes a las medidas, acciones y políticas estatales adoptadas de los datos diarios vinculados a esta pandemia, incluyendo la imagen del tablero operacional mencionado.

En este sentido, esta estrategia comunicacional del Estado uruguayo ha sido objeto de múltiples análisis en fuentes periodísticas internacionales como la BBC (BBC, 2020).

Dicho tablero operacional se ha convertido en la referencia nacional de la población y los medios de comunicación para obtener información sobre la

2 Tablero del Sistema Nacional de Emergencias de Uruguay, https://www.gub.uy/sistema-nacionalemergencias/pagina-embebida/visualizador-casos-coronavirus-covid-19-uruguay. 


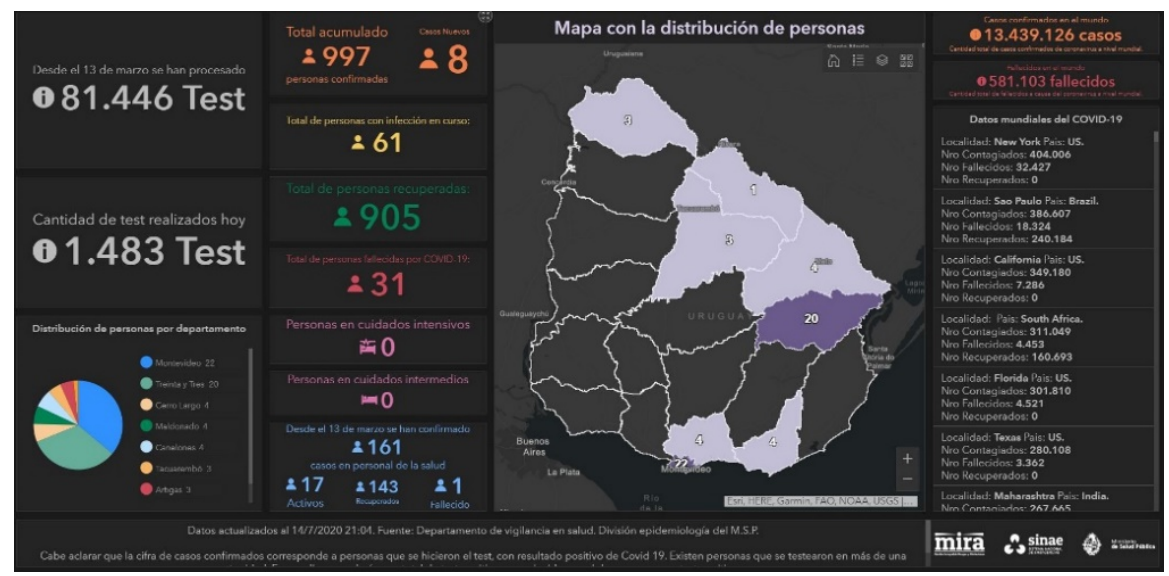

Figura 1. Tablero Operacional de Casos Oficiales de COVID-19 para la República Oriental del Uruguay para el 15 de julio de 2020.

Fuente: SINAE, 2020.

cantidad de casos activos, recuperados y fallecidos de COVID-19 a nivel nacional y departamental. Así como para consultar la cantidad de testeos realizados diariamente,cantidad de pacientes positivos internados en cuidados intensivos, personal de salud contagiado y los datos a nivel mundial y de diferentes países. De acuerdo con la información suministrada por SINAE, dicha herramienta tiene del orden de 200.000 visitas diarias y más de 18 millones y medio de visitas acumuladas desde sus inicios (del 9 de abril al 15 de julio de 2020), tal como se presenta en la gráfica de la Figura 2.

En lo que refiere al uso de los datos geoespaciales para la gestión de la pandemia, se destaca su aplicación a nivel interno del SINAE como apoyo a la toma de decisiones del gobierno nacional, mediante el Monitor Integral de Riesgos y Afectaciones (MIRA). ${ }^{3}$ Herramienta que es utilizada para el cruzamiento espacial de datos demográficos, datos de salud (como ser disponibilidad de camas en CTI y localización de los centros asistenciales), información sobre zonas vulnerables, con los datos de localización de los casos positivos. Análisis que permite realizar un seguimiento detallado de dichos casos e identificar posibles focos de contagio. Todos ellos son aspectos altamente relevantes para enfrentar esta pandemia y que posibilitan la toma de decisiones y formulación de acciones oportunas (VTV, 2020). 


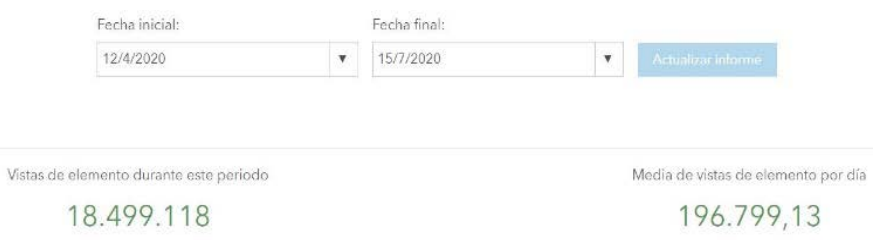

Serie de tiempo de uso

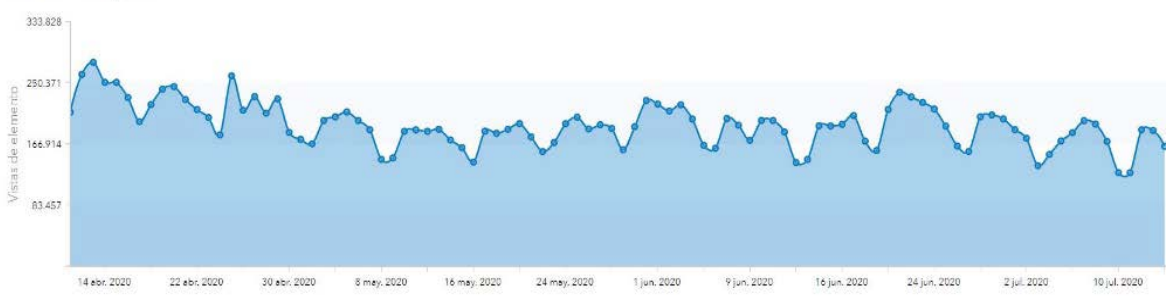

Figura 2. Estadísticas de acceso al Tablero Operacional de Casos de Coronavirus en el Uruguay para el 15 de julio de 2020.

Fuente: SINAE, 2020.

Complementariamente, como herramienta de seguimiento de casos se ha desarrollado una aplicación móvil, llamada "CoronavirusUY", realizada bajo la coordinación de la Agencia de Gobierno Electrónico y Sociedad de la Información y Conocimiento (AGESIC). ${ }^{4}$ En su última actualización se incorpora la tecnología de alerta de proximidad con personas contagiadas, desarrollada por Apple y Google (Google, 2020).

Dicha aplicación ha cobrado gran interés por la población ya que cuenta con más de 421000 descargas hasta el 22 de julio de 2020, según información aportada en medios de prensa nacionales (De Marco, 2020).

Para la población total del Uruguay, que es del orden de los 3.5 millones de habitantes, los números presentados son elevados y muestran, de alguna manera, una apertura de la ciudadanía a la utilización de herramientas y aplicaciones geomáticas para combatir el COVID-19.

\section{Limitantes técnicas y formales para un mejor aprovechamiento}

Ahora bien, cuando se quiere profundizar en el uso de la geomática para tareas de análisis espacial (como ser el cruce de variables geográficas para encontrar eventuales causas o analizar escenarios posibles), que es su gran diferencial, surgen algunas limitantes.

4 Recuperado de: https://www.gub.uy/agencia-gobierno-electronico-sociedad-informacionconocimiento/ 
Muchos de los datos (o geodatos) que se consideran útiles para profundizar en los análisis de cara a una mejor gestión territorial, presentan calidades no del todo adecuadas, ya sea por su exactitud geográfica, resolución temporal o linaje.

En el caso de Uruguay, en este aspecto sobresale la necesidad de contar con datos sociodemográficos actualizados que, si bien están georreferenciados, fueron relevados en el año 2011 en el Censo Nacional de Población y Vivienda realizado por el Instituto Nacional de Estadísticas (INE). Esta desactualización limita las posibilidades del análisis geomático ya que la toma de decisiones y elaboración de políticas basadas en estos pueden atentar contra la eficiencia de las acciones y planes que se propongan. Asimismo, como el INE no publica dichos datos en formato geo-amigable, se requiere la realización de procesos previos por parte de los usuarios, para que puedan ser utilizados análisis espaciales.

Si bien existen algunos otros datos geoespaciales, como ser las redes de infraestructura (agua potable, saneamiento, entre otros), los locales de enseñanza, etc., que están disponibles al público, otros muchos no lo están. Lo que se agrava con el hecho de que no todos estos datos son directamente integrables en un proyecto de análisis espacio-temporal.

Por otro lado, la discusión académica sobre la utilización de datos privados, que involucra la componente espacial, para la lucha contra el COVID-19 ya se está dando en Uruguay. En un artículo publicado recientemente, Gustavo Betarte y otros investigadores de la Universidad de la República analizan los desafíos de seguridad y privacidad en la implementación de soluciones de rastreo de proximidad. Dicha tecnología ya se encuentra en uso en el país a través de la última actualización de la aplicación Coronavirus UY. ${ }^{5}$ En este desarrollo se solicita a los usuarios su autorización para realizar el uso de los datos capturados por sus dispositivos móviles para el envío o recepción anónima ${ }^{6}$ de códigos. Adicionalmente, se preserva la privacidad e identidad tanto de quienes la usan como de quienes autorizan la comunicación anónima de su contagio.

Dando cumplimiento con la normativa internacional y nacional vigente, el dato de localización del individuo es información que debe ser protegida. El derecho a la privacidad es reconocido en el mundo entero y a nivel global y está considerado en diversos acuerdos internacionales como en la Declaratoria Universal de los Derechos Humanos. ${ }^{7}$ A nivel país, además de la aplicación de dichas normas

5 Aplicación CoronavirusUY: https://www.presidencia.gub.uy/comunicacion/comunicacionnoticias/ coronavirus-aplicacion-alerta-de-exposicion

6 Anonimato que se maximiza con la generación de códigos aleatorios que cambian cada 15 minutos.

7 Acuerdos como ser: Reglamento Europeo № 2016/679; Convenio № 108 del Consejo de Europa y su protocolo de noviembre de 2001; Estándares en Protección de Datos Personales para los Estados Iberoamericanos emitidos por la Red Iberoamericana de Protección de Datos de junio de 2017; 
internacionales se cuenta con normativas nacionales ${ }^{8}$ que velan por la privacidad de los datos. Protección que se alinea con la visión sostenida por el Estado uruguayo, en el combate al COVID-19, de preservar la información personal brindando solamente datos generales de los casos y con un nivel mínimo de desagregación. En este sentido, en abril del 2020, y en virtud de la Emergencia sanitaria, la Unidad Reguladora y de Control de los Datos Personales (URCDP) elaboró un documento ${ }^{9}$ que brinda recomendaciones para personas y entidades públicas o privadas que trabajen con datos personales asociados al coronavirus COVID-19. El documento contiene información sobre los requisitos para el tratamiento de datos sensibles, quienes son los sujetos legitimados para el tratamiento de datos de salud, consentimiento del titular, principios generales de la Protección de Datos Personales y enlaces de interés sobre el marco jurídico nacional vigente en la materia, entre otros. Asimismo, el texto recuerda que el empleo de información personal se encuentra alcanzado por normas que reconocen el derecho a la Protección de Datos Personales como un derecho fundamental y fomenta el uso responsable de dicha información procurando alcanzar su equilibrio con otros derechos.

Consecuentemente, estos principios ocasionan, en cierta medida, otra limitante que refiere a la existencia de restricciones formales de acceso a los datos primarios. A modo de ejemplo, el Ministerio de Salud Pública no especifica en qué ciudades, barrios o manzanas se detectan los casos de las personas diagnosticadas positivas de COVID-19, sino que la información pública llega solamente a nivel de los departamentos (división administrativa mayor). Es decir, el nivel de desagregación permitido es muy bajo para el caso de análisis territoriales de detalle. Esto quedó en evidencia para el Departamento de Geomática del Instituto de Agrimensura de la Facultad de Ingeniería de la Universidad de la República, cuando se propuso replicar una investigación extranjera referida al estudio de factores determinantes para la propagación del COVID-19 y requirió disponer de geodatos nacionales que no estaban accesibles. Además, se enfrentó con la dificultad, no menor, referida al escaso nivel de detalle y desactualización de la información. La ausencia de accesibilidad a datos adecuados es una limitante que restringe el

Protocolo de Modernización del citado Convenio aprobado por el Comité de Ministros del Consejo de Europa el 18 de mayo de 2018.

8 En Uruguay las siguientes normativas protegen los datos personales: Ley $\mathrm{N}^{\circ} 18.331$ de 11 de agosto de 2008; Ley $N^{\circ} 19.670$ de 15 de octubre de 2018; Decreto $N^{\circ}$ 414/009 de 31 de agosto de 2009 (que viene a reglamentar algunos aspectos de la ley 18.331); el Decreto $\mathrm{N}^{\circ} 64 / 020$ de 17 de febrero de 2020 (que viene a reglamentar varios artículos de la ley $\mathrm{N}^{\circ} 19.670$ y un artículo de la ley $\mathrm{N}^{\circ} 18.331$ ).

9 Recuperado de https://www.gub.uy/unidad-reguladora-control-datos-personales/comunicacion/ noticias/recomendaciones-para-tratamiento-datos-personales-ante-situacion-emergencia 
conocimiento y dimensionamiento real de la pandemia, así como la realización de investigaciones relevantes y complementarias (Rolnik, 2020).

\section{Riesgos de la "nueva" exposición de la información geoespacial}

Esta "repentina" visibilidad que ha cobrado el uso de la información geoespacial presenta ciertos riesgos, que se han identificado en una lista no exhaustiva ni ordenada por importancia, la cual detalla a continuación:

- Abuso por parte de los propietarios de los datos geoespaciales. El uso de la información geoespacial para gestionar la pandemia y su frágil límite con la privacidad individual es objeto de intensos debates, a nivel ideológico y legal, que podrían desembocar en una percepción negativa de la geomática al no quedar claramente expuesto el límite entre los aspectos éticos y técnicos.

Muchos científicos manifiestan su preocupación sobre el hecho de que, a través de la búsqueda de respuestas para gestionar situaciones de emergencia sanitaria, puedan surgir solución+es que resulten en sistemas que permitan una bio-vigilancia estricta de la sociedad en general. En el caso de algunos países asiáticos, el énfasis, para la detección y el seguimiento de casos positivos de COVID-19, está puesto en la detección individual mediante la vigilancia digital constante y estricta de las personas contagiadas, a través de sus dispositivos personales. El uso de los teléfonos móviles y las tarjetas de crédito pasarían a ser instrumentos de control que permiten trazar los recorridos de sus portadores.

La geomática podría interpretarse, entonces, como una tecnología que "invade" la privacidad de las personas, publicando o dotando a los propietarios de dichas herramientas de información privilegiada, como ser los domicilios, datos la movilidad y costumbres de los individuos (Preciado, 2020).

- Desaprovechamiento de la oportunidad para cobrar relevancia más allá de lo comunicativo. Sin dejar de apreciar el valor que tiene la geomática como herramienta de comunicación, el riesgo de que su aporte se limite a ser "ese mapita digital que aparece en la televisión" ahora devenido un tablero de control geográfico, es evidente (Buzai, 2020).

La geomática y los sistemas de información geográfica permiten modelar, mediante el uso de datos geoespaciales específicos, una situación particular del territorio en estudio. Por ello, la calidad de la cartografía resultante de las predicciones generadas y de los resultados obtenidos de las aplicaciones realizadas sobre ellos, dependen de los datos ingresados y de la pertinencia de los procesos desarrollados.

En este sentido, la comunidad geomática debe jugar un rol destacado para promover y crear conciencia sobre la importancia de contar con datos actualizados y de buena calidad, así como incentivar la formación de recursos 
humanos en estas áreas. Sería un paso atrás en el futuro de la información geográfica, que la mirada de los gobernantes y de la sociedad en general, quede restringida a la comunicacional. En otras palabras, que sólo se aprecie el potencial semiótico de las herramientas existentes, perdiéndose su capital fundamental: la potencia de captura, procesamiento, análisis y simulación de la información espacio-temporal (Dangermond et al., 2020).

- Sobrevaloración de la geomática como LA herramienta de combate al COVID19. Las herramientas geomáticas, para dar respuesta a situaciones de emergencia, principalmente sanitarias, han sido ampliamente usadas durante mucho tiempo. Aplicaciones que se han utilizado en la creación de mapas basados en análisis espaciales, para la construcción de modelos para realizar simulaciones y predicciones, así como para elaborar soluciones y guiar los relevamientos de datos. (Dangermond et al., 2020).

Pero, es evidente que la geomática es "UNA" entre un conjunto de herramientas para el combate al COVID-19. No es la única y tampoco es la más importante.

Por lo tanto, la tentación de pensar a la geomática como la mejor opción del combate a la pandemia es un gran riesgo. Estas exageraciones son frecuentes en los profesionales de cada rubro y, principalmente, en las empresas que venden soluciones geoinformáticas, etc.

\section{Oportunidades de mejora}

Como puede apreciarse, la geomática ha cobrado inesperadamente una relevancia general inusitada pero positiva. Este hecho presenta, dadas las limitaciones y los riesgos descritos, grandes desafíos para la comunidad de profesionales y académicos que la integran. En este sentido, se enumeran algunas oportunidades de mejora de ciertos aspectos que pueden aportar a un salto de calidad en el posicionamiento de la geomática, en sus métodos, tecnologías y herramientas así como en su democratización y adopción por el público en general:

- Necesidad de generación y difusión de datos adecuados. Como ya se mencionó tanto la captura, la actualización y el procesamiento básico de geodatos con estándares de calidad suficientes, se vuelve crucial para que la geomática pueda aportar, aún más en el análisis y conocimiento de situaciones con componente territorial (Boulos y Geraghty, 2020).

Para ello, es necesario que las instituciones (privadas, pero sobre todo, las públicas) entiendan que invertir en la obtención y actualización permanente de bases de datos geográficas nunca puede ser considerado un gasto sin retorno. Ejemplo de ello es el reciente "Proyecto para la generación de imágenes, 
modelos digitales y cartografía", ${ }^{10}$ coordinado por el equipo técnico de la Infraestructura de Datos Espaciales de Uruguay. ${ }^{11}$ (IDE Uy). Proyecto que permitió disponer de algunas bases cartográficas actualizadas y de imágenes digitales a nivel nacional. Hecho que si bien ha significado un gran avance en lo que refiere a disponer de datos nacionales actuales, requiere para mantener la inversión, de la realización periódica de vuelos nacionales o regionales, de similares características y no en el largo plazo. Aún mejor sería que el uso de imágenes satelitales de alta resolución, que ya se están produciendo a gran escala, fuera una política habitual de actualización de geodatos nacionales. Otra muestra de periodicidad en la captura de datos refiere a los relevamientos censales que lleva adelante el INE (institución que integra la IDE nacional) para los que sería deseable que exista una política de actualización permanente. En este sentido, el cometido de la IDE Uy de liderar la articulación y el fortalecimiento de la producción cartográfica, así como que el acceso de la información geográfica fiable y oportuna cobra un rol protagónico e imprescindible.

- Accesibilidad a geodatos simplificada. Es necesario avanzar en la simplificación del acceso a los geodatos, no alcanza con que los mismos estén capturados y actualizados, sino que, su disponibilidad sea tan importante como lo anterior. El país ha avanzado en este punto, pero aún falta mucho camino por recorrer. En este sentido, se ve como uno de los puntos clave el desarrollo y avance de geoservicios con los estándares que se establezcan.

Una de las ventajas o consecuencias directas de este modelo, es que el control de calidad de los geodatos se hace de manera "cruzada" entre los diferentes organismos que participan de la iniciativa. Cada uno de ellos es responsable de la información que produce y, a su vez, puede controlar y ser controlado por los demás, en cuanto a la calidad de la información geoespacial que produce y publica. Este hecho, lejos de ser una amenaza, es en realidad, una gran oportunidad para la comunidad de usuarios y la sociedad en general, ya que es el sistema en su totalidad quien se ve favorecido por una mejora general en la

Recuperado de https://www.gub.uy/infraestructura-datos-espaciales/comunicacion/noticias/ presentacion-del-geoportal-ideuy

De acuerdo a la página oficial de la IDE: "La Infraestructura de Datos Espaciales fue creada por los Art. 35 y 36 de la Ley 19.149 de 2013 como un órgano desconcentrado de Presidencia de la República, con autonomía técnica. Su cometido es liderar la articulación y el fortalecimiento de la producción y el acceso de la información geográfica del Uruguay para que sea fiable, oportuna, interoperable, de alta calidad, y brinde apoyo en la toma de decisiones para el desarrollo nacional; esto incluye a organismos públicos, academia, empresas y ciudadanos. Se inspira en los principios de cooperación y coordinación entre las administraciones, así como en la transparencia y el acceso a la información pública." Recuperado de https://www.gub.uy/infraestructura-datosespaciales/institucional/creacion-evolucion-historica 
calidad de la información geográfica y, en última instancia, por las decisiones que se tomen a partir de dichos datos.

- Posicionamiento de la geomática como herramienta indispensable de gestión para las autoridades. Parece notarse una mejora en la valoración que las autoridades nacionales tienen sobre las herramientas geomáticas, pero este hecho no debe sólo circunscribirse al aspecto de la comunicación. Hace falta que los gobernantes entiendan y valoren además y, principalmente, las capacidades de análisis que la geomática permite, a partir de buenos datos, buena tecnología y buenas prácticas, todas bien combinadas con buenos recursos humanos. (CEPAL, 2020). A la capacidad de análisis le podemos agregar dos perspectivas nuevas: la capacidad de simulación a partir de datos reales y la capacidad de analizar enormes volúmenes de datos geográficos, esto último conocido como el "big data geográfico".

El gran desafío, que aún persiste, es el reconocimiento de la geomática como herramienta indispensable para el apoyo a la toma de decisiones de políticas, tanto del sector público como privado, que va más allá de situaciones de riesgo como es la pandemia del COVID-19. Incluso, algunos autores levantan la bandera del derecho de todos los ciudadanos a ser geo-informados y que el derecho a la geoinformación debe ser el principio directriz de las autoridades geográficas (Ugeda, 2020).

Finalmente, es importante destacar que, si bien el uso de los datos y las tecnologías geoespaciales han jugado un papel muy importante como respuesta a la pandemia, su aporte no ha finalizado, ya que cobrará un rol primordial a medida que se vuelva a "abrir" el mundo (Data, 2020). Se debe seguir avanzando y pasar de la respuesta a la recuperación y después a la reapertura, para lo cual la geomática tiene mucho para aportar, ya sea representando tendencias, resiliencia comunitaria o analizando diversos impactos y riesgos (Geraghty, 2020).

\section{Referencias}

BBC News Mundo (29 de mayo de 2020) "Coronavirus en Uruguay: la singular y exitosa estrategia del país para contener la pandemia sin cuarentena obligatoria”. Recuperado de https://www.bbc.com/mundo/noticias-america-latina-52837193,

Betarte, G.; Campo, J.; Delgado, A.; Ezzati, P.; Forteza, A.; Gonzalez, L.; Martín, A.; Muracciole, B. \& Ruggia, R. (2020). Desafíos de seguridad y privacidad en el diseño e implementación de soluciones de rastreo de proximidad. 11 pp., UDELAR -PEDECIBA, Montevideo.

Boulos M. N., \& Geraghty E. M. (2020). Geographical Tracking and Mapping of Coronavirus Disease COVID-19/severe Acute Respiratory Syndrome Coronavirus 2 (SARS-CoV-2) 
Epidemic and Associated Events Around the World: How 21st Century GIS Technologies Are Supporting the Global Fight Against Outbreaks and Epidemics. International Journal of Health Geographics, 19, 8, 2020.

Buzai, G. D. (2020). De Wuhan a Luján. Evolución espacial del COVID-19. Revista Posición, 3.

CEPAL (2020). Las oportunidades de la digitalización en América Latina frente al COVID-19.

Dangermond, J.; De Vito, C., \& Pesaresi, C. (2020). Using GIS in the Time of the COVID-19 Crisis, casting a glance at the future. A joint discussion. J-Reading-Journal of Research and Didactics in Geography, vol. 1.

Datta, A. (8 de junio de 2020). "Why geospatial technologies will be at forefront as the world reopens" [Mensaje en un blog]. Geospatial World Blog. Recuperado de https://www.geospatialworld.net/blogs/

De Marco, J. P. (22 de julio de 2020). Ningún uruguayo recibió alertas de exposición en Coronavirus UY luego de 36 días de funcionamiento. Diario El Observador, Uruguay. Recuperado de: https://www.elobservador.com.uy/nota/ningun-uruguayo-recibioalertas-de-exposicion-en-coronavirus-uy-tras-36-dias-de-funcionamiento20207211830

Geraghty, E. (2 de junio de 2020). "COVID-19: Five Spatial Approaches to Safely Reopen" [Mensaje en un blog]. ESRI Blog. Recuperado de: https://www.esri.com/about/newsroom/blog/

Google (2020). Notificaciones de exposición: cómo usar la tecnología para ayudar a las autoridades de salud pública a combatir el COVID-19. Recuperado de https://www.google.com/covid19/exposurenotifications/

La República (15 de junio de 2020). "Paganini: Coronavirus Uy coloca a Uruguay 'a la vanguardia'”, Uruguay). Recuperado de https://www.republica.com.uy/paganini-coronavirus-uy-coloca-a-uruguay-a-la-vanguardia-id770656/

Montaner, C. (22 de mayo de 2020). "Coronavirus: Uruguay, el oasis de la pandemia en América del Sur”. CNN Opiniones. Recuperado de

https://cnnespanol.cnn.com/2020/05/22/opinion-coronavirus-uruguay-el-oasis-dela-pandemia-en-america-del-sur/

Preciado, P. (28 de marzo de 2020). Aprendiendo del virus: La gestión política de las epidemias pone en escena la utopía de comunidad y las fantasías inmunitarias de una sociedad, externalizando sus sueños de omnipotencia de su soberanía política. Diario El País, España. Recuperado de https://elpais.com/elpais/2020/03/27/opinion/1585316952_026489.html

Rolnik, R. (4 de junio de 2020). "Não se enfrenta a pandemia com leituras simplificadas do território" [Mensaje en un blog] Cidade é Nossa. Recuperado de https://raquelrolnik.blogosfera.uol.com.br/2020/06/04/nao-se-enfrenta-apandemia-com-leituras-simplificadas-do-territorio/

Tribollet, A. (15 de julio de 2020) "The Role of GIS during a Pandemic: Why the "Where" Is So Important for the Coronavirus" UTAH AGRC. Recuperado de: https://gis.utah.gov/ the-role-of-gis-during-a-pandemic-why-the-where-is-so-important-for-thecoronavirus/ 
Ugeda, L. (15 de julio de 2020). "A covid-19 acelera o diretito à geoinformação" Diario: Observador (Portugal). Recuperado de https://observador.pt/opiniao/a-covid-19-acelera-o-direito-a-geoinformacao/

VTV Noticias (25 de mayo de 2020). "Informe especial: cómo funciona el Monitor Integral de Riesgos y Afectaciones”. [Archivo de video]. Recuperado de https://www.youtube.com/watch?v=_LWY0jcNk28\&feature=youtu.be 